\title{
Predicting Functional Brain ROIs via Fiber Shape Models
}

\author{
Tuo Zhang ${ }^{1,2, *}$, Lei Guo ${ }^{1}$, Kaiming $\mathrm{Li}^{1,2}$, Dajing $\mathrm{Zhu}^{2}$, \\ Guangbin $\mathrm{Cui}^{3}$, and Tianming $\mathrm{Liu}^{2}$ \\ ${ }^{1}$ School of Automation, Northwestern Polytechnical University, Xi' an, China \\ ${ }^{2}$ Department of Computer Science and Bioimaging Research Center \\ The University of Georgia, Athens, GA, USA \\ ${ }^{3}$ Department of Radiology, Tangdu Hospital, Xi' an, China
}

\begin{abstract}
Study of structural and functional connectivities of the human brain has received significant interest and effort recently. A fundamental question arises when attempting to measure the structural and/or functional connectivities of specific brain networks: how to best identify possible Regions of Interests (ROIs)? In this paper, we present a novel ROI prediction framework that localizes ROIs in individual brains based on learned fiber shape models from multimodal task-based fMRI and diffusion tensor imaging (DTI) data. In the training stage, ROIs are identified as activation peaks in task-based fMRI data. Then, shape models of white matter fibers emanating from these functional ROIs are learned. In addition, ROIs' location distribution model is learned to be used as an anatomical constraint. In the prediction stage, functional ROIs are predicted in individual brains based on DTI data. The ROI prediction is formulated and solved as an energy minimization problem, in which the two learned models are used as energy terms. Our experiment results show that the average ROI prediction error is $3.45 \mathrm{~mm}$, in comparison with the benchmark data provided by working memory task-based fMRI. Promising results were also obtained on the ADNI-2 longitudinal DTI dataset.
\end{abstract}

Keywords: diffusion tensor imaging, fMRI, working memory, fiber shape model.

\section{Introduction}

Mapping of structural and functional connectivities of the human brain via neuroimaging offers an exciting and unique opportunity to understand the brain architecture, and thus has received significant interest [1-5]. However, a fundamental question arises when attempting to map structural and functional connectivities: how to define the best possible Regions of Interests (ROIs) for the connectivity mapping? Essentially, ROIs provide the structural substrates for measuring connectivities within individual brains and for pooling data across population groups. Thus, identification of reliable, reproducible and accurate ROIs is critically important for the success of

\footnotetext{
* Tuo Zhang was supported by Doctorate Foundation of Northwestern Polytechnical University.
} 
connectivity mapping. However, in our view, this task is challenging due to the unclear boundaries between cortical regions, remarkable variability of brain anatomy, and nonlinearity of ROIs. For instance, a slight change of the shape, size or location of a ROI might dramatically alter its structural and functional connectivity profiles [6].

In this paper, we present a novel framework that learns fiber shape model and anatomical constraint model of functional ROIs based on multimodal task-based fMRI and DTI data in the training stage, and apply the predictive models to localize functional ROIs in testing samples based only on DTI data. The major advantages of this framework and our contributions lie in the following two aspects. 1) In the training stage, the activated brain regions derived from task-based fMRI data provide the benchmark ROI data for learning prior shape models of white matter fibers emanating from these ROIs. Recent literature using working memory task-based fMRI data [6] demonstrated that the fiber connection patterns of corresponding functional ROIs are quite consistent, providing direct evidence that white matter fiber connection pattern is a good predictor of functional landmark [7]. This is similar to the "connectional fingerprint" concept presented in [8]. 2) In the prediction stage, only DTI data is needed to accurately localize the functional ROIs based on our predictive models, without relying on the availability of task-based fMRI data. Typically, a DTI scan needs less than ten minutes, is much less demanding, and is widely available. Therefore, functional brain ROIs prediction based on DTI has wide applications in brain imaging.

\section{Materials and Methods}

The flowchart of our ROI prediction framework is illustrated in Fig. 1. It consists of two stages: model training and ROI prediction. The model training (the purple box in Fig.1) is conducted on the training dataset in which each subject has both task-based fMRI and DTI data. Then, descriptive shape features of fiber bundles were extracted, and fiber shape models and ROIs location distribution models are learnt. In the prediction stage (the green box in Fig. 1), the learned predictive models are applied on subjects with DTI data by minimizing an energy function to obtain the predicted ROIs. Finally, we use taskbased fMRI data to validate the prediction result (black dashed box).

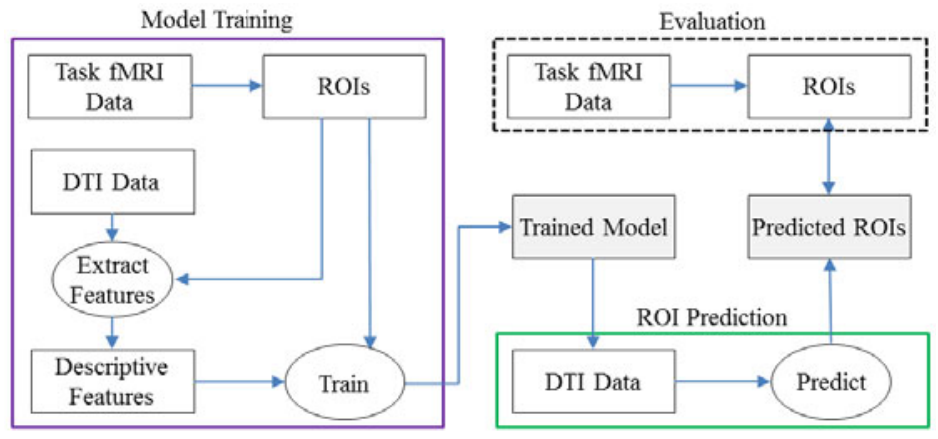

Fig. 1. The flowchart of the model training and ROI prediction framework 


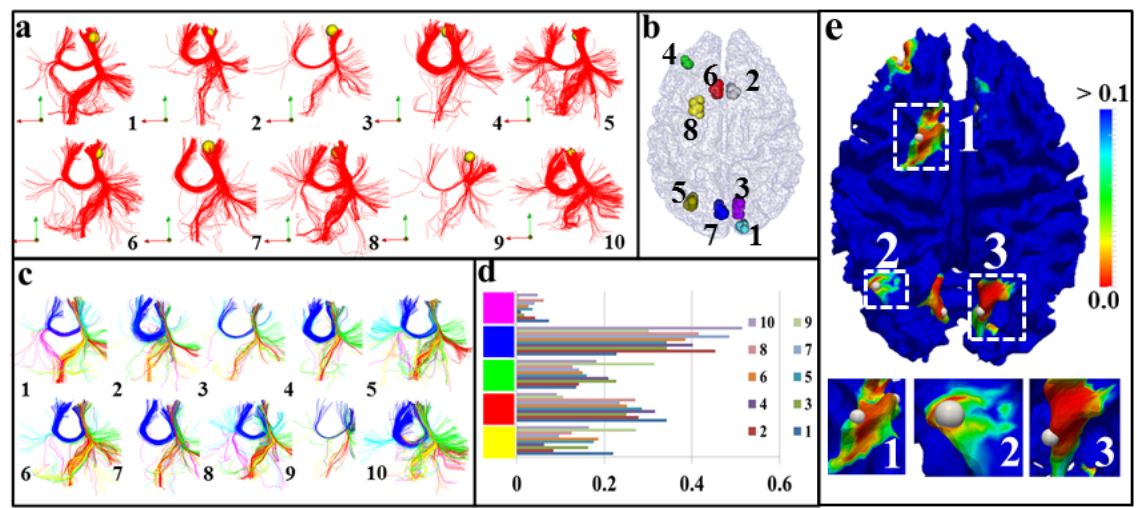

Fig. 2. (a) Fiber bundles extracted for ROI \#8 of the training dataset; (b) Examples of consistent ROI locations with ROI IDs labeled. The corresponding ROIs are coded with the same color; (c) Clustered fiber bundles in (a); (d) Shape histograms of fiber bundles in (c). Shape pattern on the vertical axis is color-coded the same way as that in (c); (e) Convergence basins of the energy function Eq. (1) in Section 2.3. More details are referred to the text.

\subsection{Data Acquisition and Pre-processing}

Fifteen healthy young adults voluntarily participated in this study. Each volunteer performed a modified version of the OSPAN working memory task (3 blocks: OSPAN, Arithmetic and Baseline) while fMRI data was acquired. DTI scans were also obtained for each volunteer. Briefly, both fMRI and DTI scans were acquired on a 3T GE Signa scanner with acquisition parameters as follows: fMRI: $64 \times 64$ matrix, 4mm slice thickness, 220mm FOV, 30 slices, TR $=1.5 \mathrm{~s}$, TE $=25 \mathrm{~ms}$, ASSET $=2$; DTI: $128 \times 128$ matrix, $2 \mathrm{~mm}$ slice thickness, $256 \mathrm{~mm}$ FOV, 60 slices, TR $=15100 \mathrm{~ms}$, ASSET $=2,30$ gradient directions, $\mathrm{b}$-value $=1000$. The pre-processing of the DTI data includes brain skull removal and motion correction. Afterwards, the diffusion tensor was computed and the fiber was tracked via the MEDINRIA toolkit. The fMRI data of the OSPAN task was analyzed using the FSL FEAT. Individual activation map reflecting the OSPAN (complex span) contrast was identified. For each subject, the DTI space is used as the standard space, in which the gray matter (GM)/white matter (WM) surface is generated via an approach similar to that in [9]. The cortical surface was used as the ROI definition and prediction space. Co-registration between DTI and fMRI data is performed using FSL FLIRT. Currently, 8 most consistently activated working memory ROIs including 1. Left Occipital Pole, 2. Left Paracingulate Gyrus, 3. Left Precuneus, 4. Right Dorsolateral Prefrontal Cortex, 5. Right Lateral Occipital Gyrus, 6. Right Paracingulate Gyrus, 7. Right Precuneus, and 8. Right Superior Frontal Gyrus, were used for model training and ROI prediction. We divided the dataset into two subsets: the training dataset containing ten subjects, and the testing datasets containing the rest five subjects. A randomly selected subject from the training dataset was used as the template onto which all the other subjects were linearly registered. 


\subsection{Model Training}

The main idea of model training is to extract descriptive structural and anatomic features from the activated ROIs, and then learn predictive models based on these features. The structural features are the fiber connection profiles of the activated ROIs, and we propose a fiber shape pattern histogram PCA (principal component analysis) model to represent the fiber connection information for each ROI. The descriptive anatomical features are the spatial location distribution of the activated ROIs, and we propose an ROI location distribution PCA model.

Fiber Shape Pattern Histogram PCA Model. The fiber shape pattern histogram PCA model is constructed for each ROI separately across the training subjects to embed group-wise fiber shape information. Taking ROI $i$ for example, we define $R_{j}^{i}$ as the local region centered at location of ROI $i$ on subject $\mathrm{j}$ 's surface, and fiber bundle penetrating $R_{j}^{i}$ was extracted and denoted as $F_{j}^{i}$. We defined $T_{i}=\left\{F_{j}^{i} \mid j=\right.$ $1, \cdots, m\}$ as the fiber bundle set of ROI $i$, where $m$ is the number of the training subjects. As an example, Fig. 2a shows fiber bundles of $T_{8}$, from which consistence of connection pattern of fiber bundles can be observed. As fiber shape is considered as an effective descriptive feature of structural connectivity [10], we constructed a fiber shape pattern histogram PCA model $H_{i}$ for ROI $\mathrm{i}$ on $T_{i}$ as follows. First, we conducted a fiber shape clustering via the method in [10] on fiber tracts of the entire training dataset and clustered all fiber tracts into five shape patterns. Then for fiber bundle $F_{j}^{i}$, we define the normalized shape pattern histogram $\mathrm{h}_{j}^{i}$, in which each bin denotes the ratio of fiber tract number of each shape pattern to total fiber tract number in $F_{j}^{i}$. In Fig. 2c, we illustrate the clustered fiber bundles of Fig. 2a, and their corresponding normalized shape pattern histograms are shown in Fig. 2d, from which the consistency of $\left\{\mathrm{h}_{j}^{i} \mid j=1, \cdots, m\right\}$ can be visually observed. Afterwards, by taking $\mathrm{h}_{j}^{i}$ as the descriptive feature of $F_{j}^{i}$, we embed the shape information across the training datasets by applying PCA on the feature matrix $\left[h_{1}^{i}, h_{2}^{i}, \cdots, h_{m}^{i}\right]^{\mathrm{T}}$. The obtained mean feature $h_{\text {mean }}^{i}$ and eigen vectors, PCA transformation matrix, $E_{h}^{i}$ compose the fiber shape pattern model for ROI i, denoted as $H_{i}=\left\{h_{\text {mean }}^{i}, E_{h}^{i}\right\}$. It is noteworthy that, for each ROI, we computed the ratio between the first eigen value and the sum of all eigen values. The ratio is as high as $0.88 \pm 0.13$, suggesting the consistency in $T_{i}$ across subjects and the effectiveness of feature $\mathrm{h}_{j}^{i}$ and model $H_{i}$.

ROI Location Distribution PCA Model. Despite its variation, the spatial distribution patterns of the ROIs in the template space have certain degree of consistency, reflecting the existence of common human brain anatomical architecture (Fig. 2b), and therefore they were captured and modeled across training subjects. For subject $\mathrm{j}$, we define ROI coordinates vector $d_{j}=\left[\mathrm{x}_{1}, \mathrm{y}_{1}, z_{1}, \cdots, \mathrm{x}_{n}, \mathrm{y}_{n}, z_{n}\right]^{T}$ to be the feature describing the ROI location distribution, where $\mathrm{n}$ denotes the number of ROIs. Similarly, a PCA model $D=\left\{d_{\text {mean }}, E_{d}\right\}$ was computed on feature matrix $\left[d_{1}, \cdots, d_{m}\right]^{T}$ and the first eigenvalue ratio is 0.72 , suggesting the consistency of $d_{j}$ and the effectiveness of PCA model. It is noted that fiber shape pattern histogram PCA model $H_{i}$ represent structural connectivity feature for each ROI individually, while ROI location PCA model $D$ represent the ROI spatial distribution in the entire training dataset. 


\subsection{ROI Prediction Framework}

ROI prediction was conducted only based on DTI data of an individual subject and the trained models. The prediction was formulated as an energy function minimization problem by maximally mapping the trained models onto the DTI data of the subject being predicted. The energy function is defined as:

$$
E=\lambda E_{\text {int }}+(1-\lambda) E_{\text {ext }}
$$

where $E_{\text {ext }}$ denotes the mapping of fiber shape pattern histogram PCA model, while $E_{\text {int }}$ is the ROI location PCA model constraint, and ë trades off between them.

Similar to section 2.2, let $\tilde{d}=\left[\mathrm{x}_{1}, \mathrm{y}_{1}, z_{1}, \cdots, \mathrm{x}_{n}, \mathrm{y}_{n}, z_{n}\right]^{T}$ be the location of ROIs being predicted on the cortical surface. For each ROI, fiber bundles $\tilde{F}^{i}$ were extracted from local region $R^{i}$, and the corresponding shape histogram $\tilde{h}^{i}$ were obtained.

To derive $E_{\text {ext }}$, we mapped $\tilde{h}^{i}$ into PCA model $H_{i}=\left\{h_{\text {mean }}^{i}, E_{h}^{i}\right\}$ and reconstructed a new histogram as follows:

$$
\tilde{h}_{\text {rec }}^{i}=h_{\text {mean }}^{i}+E_{h}^{i^{T}} E_{h}^{i}\left(\tilde{h}_{\text {rec }}^{i}-h_{\text {mean }}^{i}\right)
$$

If $\tilde{h}^{i}$ is consistent with those $\left\{\mathrm{h}_{j}^{i} \mid j=1, \cdots, m\right\}$ in the training dataset, then the error between it and $\tilde{h}_{r e c}^{i}$ is expected to be small. Therefore, we define $E_{\text {ext }}$ to be the sum of the errors of all ROIs:

$$
E_{\text {ext }}=\frac{1}{n} \sum_{i=1}^{n} \chi^{2}\left(\tilde{h}^{i}, \tilde{h}_{\text {rec }}^{i}\right)
$$

where $\div^{2}$ denotes $\div^{2}$-test measuring the similarity between two histograms if we let $l$ be its bin number:

$$
\chi^{2}\left(\tilde{h}^{i}, \tilde{h}_{r e c}^{i}\right)=\sum_{k=1}^{l}\left(\tilde{h}^{i}(k)-\tilde{h}_{r e c}^{i}(k)\right)^{2} /\left(\left(\tilde{h}^{i}(k)+\tilde{h}_{r e c}^{i}(k)\right)\right.
$$

$E_{\text {int }}$ can be defined as the Euclidean distance between location $\tilde{d}$ and the its reconstructed version $\tilde{d}_{r e c}$ from model $D$ :

$$
E_{\text {int }}=\operatorname{dist}\left(\tilde{d}, \tilde{d}_{r e c}\right)
$$

Global search was conducted to minimize the energy function. The search starts from the mean ROIs location of in training dataset and ends when the location becomes stable. It is noted that the two energy terms are normalized into $[0,1]$ prior to global search.

\section{Experimental Results}

At current stage, we did not take ROI size into consideration, therefore $\mathrm{R}^{\mathrm{i}}$ defined as uniform 3-ring vertices neighborhood on the cortical surface was used for all ROIs to extract fiber bundles. $\lambda$ was empirically assigned 0.3 . 


\subsection{Evaluation and Validation via Task-Based fMRI Data}

The activated ROI locations detected from task-based fMRI data of the five subjects in prediction dataset were used as benchmark to evaluate the prediction results. We randomly selected a subject from the prediction dataset to illustrate the prediction result.

First of all, we illustrate the effectiveness of the prediction framework in Fig. 2e where the 8 benchmark ROI locations are highlighted by white bubbles, and we computed the energy $E$ defined in section 2.3 between the benchmark ROI location and the neighbor vertices on surface around them. Energy values mapped onto the surface intuitively illustrate the convergence basins around the benchmark ROIs (see three zoomed-in basins in Fig. 2e), suggesting the convergence of our prediction framework.

Then, the prediction results of the 8 ROIs were shown in Fig. 3. It is evident that the fiber bundles emanating from the predicted ROIs are quite similar to those from the benchmark ROIs, indicating the effectiveness and accuracy of our ROI prediction framework. In addition, fiber bundles emanating from the predicted ROIs and benchmark ROIs are quite similar to those from the corresponding ROIs in the training dataset. Quantitatively, Table 1 shows the mean prediction errors, the Euclidian distances between the predicted ROIs and the benchmark ones, of the five subjects in the prediction dataset. On average, the prediction error for 8 eight ROIs over 5 subjects is $3.45 \mathrm{~mm}$. For most ROIs, the prediction errors are approximately 2 4 mm. Notably, the less accurate result on ROI \#1 reveals that the energy function may be trapped in a local minimum.

For the purpose of comparison, we used linear (via FSL FLIRT) and nonlinear registration (via the HAMMER software package [11]) methods to warp the training subjects onto the subjects to be predicted, and their functional ROIs were correspondingly warped and used as prediction results. The mean prediction errors between warped ROIs and benchmark ones in the prediction dataset are also shown in Table 1. On average, the prediction errors by FSL FLIRT and HAMMER are 5.72 $\mathrm{mm}$ and $5.53 \mathrm{~mm}$, respectively. As can be seen, our method $(3.45 \mathrm{~mm})$ significantly outperforms both of them.

\subsection{Application on ADNI-2 longitudinal DTI Data}

We applied our method on the ADNI-2 longitudinal DTI dataset under the premise that the white matter of working memory system in ADNI-2 mild cognitive impairment (MCI) patient is not distinctively different from the normal controls. Two scans of 10 MCI patients' data were obtained from the ADNI-2 project (http://adni.loni.ucla.edu/). The time interval between the two scans was around 3 months. As an example, the prediction results of one patient are shown in Fig. 4. The average distance between the predicted ROI locations of two scans is $(2.54 \pm 1.53 \mathrm{~mm})$. This relatively small distance and the high similarity of the fiber bundles (Fig. 4b) is a strong evidence of the reproducibility of our ROI prediction method. This result also suggests that there is no distinctive change in the white matter of MCI subject, at least in the working memory system. 


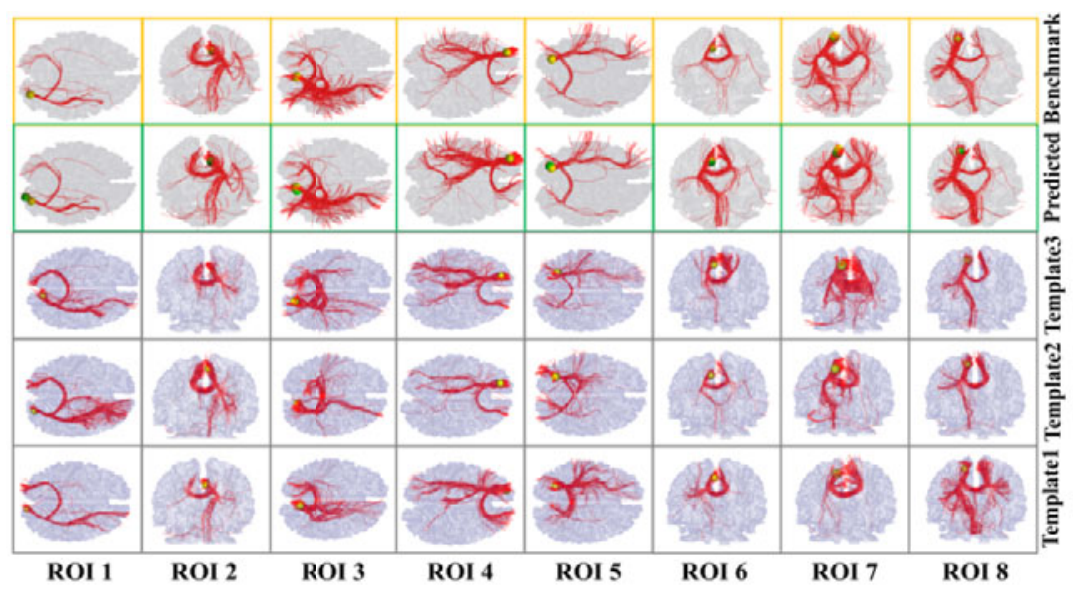

Fig. 3. Visualization of prediction results. Top yellow frame: fiber bundles emanating from benchmark ROIs (yellow bubbles); Green frame: fiber bundles emanating from predicted ROIs (green bubbles), and benchmark ROIs in the yellow frame are also displayed for comparison; Gray frames: the fiber bundles emanating from corresponding ROIs (yellow bubbles) of three subjects in the training dataset illustrated as templates for comparison.
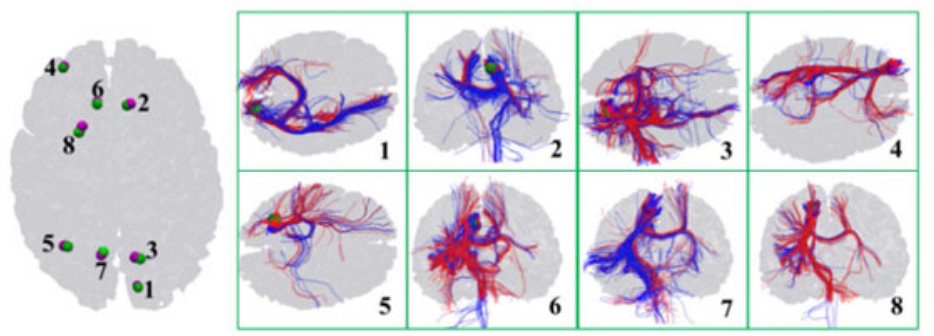

Fig. 4. Prediction results of 2 scans of ADNI- 2 subject. The predicted ROI locations of 2 scans are illustrated on the left side with ROI ID labeled. Fiber tracts extracted of the 2 scans are overlapped on the right side. (The $1^{\text {st }}$ scan: green bubbles, blue fibers; the $2^{\text {nd }}$ scan: purple bubbles, red fibers).

Table 1. Distances between the predicted ROIs and the benchmark ROIs ( $\mathrm{mm}$ )

\begin{tabular}{cccccccccc}
\hline ROIs & ROI & ROI & ROI & ROI & ROI & ROI & ROI & ROI & Mean \\
& $\mathbf{1}$ & $\mathbf{2}$ & $\mathbf{3}$ & $\mathbf{4}$ & $\mathbf{5}$ & $\mathbf{6}$ & $\mathbf{7}$ & $\mathbf{8}$ & \\
\hline Our method & 7.56 & 1.21 & 4.01 & 1.76 & 2.67 & 1.88 & 5.71 & 2.77 & $\underline{\mathbf{3 . 4 5}}$ \\
FSL FLIRT & 7.48 & 4.95 & 6.24 & 6.18 & 5.41 & 4.51 & 6.46 & 4.54 & $\underline{\mathbf{5 . 7 2}}$ \\
HAMMER & 6.18 & 4.92 & 6.40 & 7.50 & 5.15 & 3.06 & 6.08 & 4.95 & $\mathbf{5 . 5 3}$ \\
\hline
\end{tabular}

\section{Conclusion}

We presented a novel framework for functional ROI prediction using working memory network as a testing bed. Our work has demonstrated that fiber shape models 
of functional ROIs have remarkable prediction capability, providing direct support to the connectional fingerprint concept [8]. Our future work will include application and evaluation of this framework in other brain networks, and further application of this ROI prediction framework to clinical datasets such as the DTI data of ADNI-2 subjects.

\section{References}

1. Biswal, B.B., Mennes, M., Zuo, X.N., Gohel, S., Kelly, C., Smith, S.M., Beckmann, C.F., Adelstein, J.S., Buckner, R.L., Colcombe, S., Dogonowski, A.M., Ernst, M., Fair, D., Hampson, M., Hoptman, M.J., Hyde, J.S., Kiviniemi, V.J., Kötter, R., Li, S.J., Lin, C.P., Lowe, M.J., Mackay, C., Madden, D.J., Madsen, K.H., Margulies, D.S., Mayberg, H.S., McMahon, K., Monk, C.S., Mostofsky, S.H., Nagel, B.J., Pekar, J.J., Peltier, S.J., Petersen, S.E., Riedl, V., Rombouts, S.A., Rypma, B., Schlaggar, B.L., Schmidt, S., Seidler, R.D., Siegle, G.J., Sorg, C., Teng, G.J., Veijola, J., Villringer, A., Walter, M., Wang, L., Weng, X.C., Whitfield-Gabrieli, S., Williamson, P., Windischberger, C., Zang, Y.F., Zhang, H.Y., Castellanos, F.X., Milham, M.P.: Toward discovery science of human brain function. PNAS. 107(10), 4734--4739 (2010)

2. Sporns, O., Tononi, G., Kötter, R.: The human connectome: A structural description of the human brain. PLoS Comput Biol. 1(4), e42 (2005)

3. Van, Dijk. K.R., Hedden, T., Venkataraman, A., Evans, K.C., Lazar, S.W., Buckner, R.L.: Intrinsic functional connectivity as a tool for human connectomics: theory, properties, and optimization. J Neurophysiol. 103(1): 297--321 (2010)

4. Hagmann, P., Cammoun, L., Gigandet, X., Gerhard, S., Grant, P.E., Wedeen, V., Meuli, R., Thiran, J.P., Honey, C.J., Sporns, O.: MR connectomics: Principles and challenges. J Neurosci Methods. 194(1), 34--45 (2010)

5. Human Connectome Project, http://www.humanconnectomeproject.org/overview/

6. Li, K., Guo, L., Faraco, C., Zhu, D., Deng F., Zhang, T., Jiang, X., Zhang, D., Chen, H., $\mathrm{Hu}$, X., Miller, S., Liu, T.: Individualized ROI Optimization via Maximization of Groupwise Consistency of Structural and Functional Profiles. NIPS. (2010)

7. Honey, C.J., Sporns, O., Cammoun, L., Gigandet, X., Thiran, J.P., Meuli, R., Hagmann, P.: Predicting human resting-state functional connectivity from structural connectivity. PNAS. 106(6), 2035--2040 (2009)

8. Passingham, R.E., Stephan, K.E., Kötter, R.: The anatomical basis of functional localization in the cortex. Nat Rev Neurosci. 3(8), 606--616 (2002)

9. Liu, T., Li, H., Wong, K., Tarokh, A., Guo, L., Wong, S.T.: Brain Tissue Segmentation Based on DTI Data. NeuroImage. 38(1), 114--123 (2007)

10. Hu, X., Guo, L., Zhang, T., Li, G., Nie, J., Jiang, X., Zhang, D., Liu, T.: Joint analysis of fiber shape and cortical folding patterns. ISBI. (2010)

11. Shen, D., Davatzikos, C.: HAMMER: hierarchical attribute matching mechanism for elastic registration. IEEE Trans Med Imaging. 21(11), 1421--1439 (2002) 